\title{
抗肿瘤活性双四氢异喹啉生物碱类似物的合成研究进展
}

\author{
杨 扬 ${ }^{a}$ 郭 举*,a 刘站柱 $*, b$ \\ ( ${ }^{a}$ 武汉工程大学绿色化工过程教育部重点实验室＼cjkstart武汉 430205) \\ ${ }^{b}$ 中国医学科学院/北京协和医学院药物研究所 北京 100050)
}

\begin{abstract}
摘要 双四氢异喹啉天然产物是一大类具有较强生物活性的家族，目前已有近百个该类天然产物被陆续报道出来. 自 1974 年 Kluepfel 等发现了第一个具有抗肿瘤活性的四氢异哧啉天然产物以来, 已经有近百个该类四氢异喹啉天然产物 被陆续报道出来, 目前这类天然产物家族中的双四氢异喹啉生物碱因其优良的生物活性和复杂的化学结构成为了生物 学和有机化学研究领域的热点, 并进一步推动了该类天然产物家族成员 Ecteinasicdin 743 (ET-743)在欧盟成功上市, 用 于治疗晚期软组织瘤和卵巢癌. 由于包括 ET-743 在内的这类双四氢异喹啉天然产物在自然界中含量极低以及化学结 构复杂，使得对其化学结构的改造也引起了越来越多的关注. 本文基于此，综述了近十年来这类双四氢异哇啉天然产 物类似物的研究进展.
\end{abstract}

关键词＼cjkstart抗肿瘤活性；双四氢异喹啉生物碱；类似物；研究进展

\section{Progress in the Synthesis of Analogues of Bistetrahdro- isoquinoline Antitumor Alkaloids}

\author{
Yang, Yang ${ }^{a}$ Guo, $\mathrm{Ju}^{*, a} \quad$ Liu, Zhanzhu*,b
}

$\left({ }^{a}\right.$ Key Laboratory for Green Chemical Process of Ministry of Education, Wuhan Institute of Technology, Wuhan 430205)

( ${ }^{b}$ Institute of Materia Medica, China Academy of Medical Sciences \& Peking Union Medical College, Beijing 100050)

\begin{abstract}
Since the first tetrahydroisoquinoline alkaloid (THIQ), naphthyridinomycin, was found by Canadian scientist Kluepfel in 1974, nearly hundred members of this family have been reported. Such THIQ has attracted the research interest of many chemists and biologists due to its excellent biological activity and complex chemical structure. Especially, as an outstanding member of THIQ family, Ecteinascidin 743 (ET-743) has been commercialized in the European Union for the treatment of soft tissue tumors and ovarian cancer. Due to the extremely low content of natural products of bistetrahydroisoquinoline including ET-743 and the complexity of its chemical structure, the modification of its chemical structure has attracted more and more attention. Based on this, the recent advance in the synthesis of bistetrahydroisoquinoline analogues is reviewed. Keywords antitumor activity; bistetrahydroisoquinoline alkaloid; analogue; research progress
\end{abstract}

自 1974 年 Kluepfel 等 ${ }^{[1]}$ 报道了从 Streptomyces $l u$ sitanus 中分离得到了一个具有抗肿瘤活性的四氢异喹 啉天然产物 naphthriydinomycin 开始, 这类具有极强生 物活性的天然产物逐渐引起了化学家和生物学家的重 视, 目前对这类四氢异喹啉生物碱的研究重点集中于包 括 Renieramycins, Saframyeins, Jorumycins, Ecteinasicdins 等 ${ }^{[2]}$ 在内的一类双四氢异喹啉生物碱, 这类双四
氢异喹啉天然产物的结构特点是具有两个四氢异喹啉 单元, 并由两个四氢异喹啉通过哌嗪环联结成五环骨架 (图 1). 这类天然产物大多具有较强的细胞毒活性, 在抗 肿瘤和抗菌活性研究中均表现出了优良的药理活性及 独特的作用机制 ${ }^{[3 \sim 6]}$. 目前, 这类双四氢异喹啉生物碱 家族中来源于加勒比海鞘 Ecteinascidia turbinate 的 Ecteinasicdin 743 (ET-743)已先后在美国、德国和欧共体

\footnotetext{
* Corresponding authors. E-mail: guoju1984@163.com; liuzhanzhu@imm.ac.cn

Received October 30, 2018; revised January 25, 2019; published online March 29, 2019.

Project supported by the Research Fund Project of Wuhan Institute of Technology (No. K201440), the Pilot Program of Scientific Research Project of Hubei Provincial Department of Education (No. B2016057), the Open Fund of Key Laboratory of New Reactor and Green Chemical Technology of Hubei Provincial (No. 201704).

武汉工程大学科学研究基金(No. K201440)、湖北省教育厅科学研究计划指导性项目(No. B2016057)、新型反应器与绿色化学工艺湖北省重点实验室 开放基金(No. 201704)资助项目.
} 


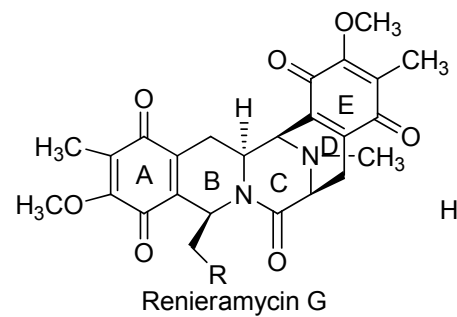

$\mathrm{R}=(E)-\mathrm{OCO}\left(\mathrm{CH}_{3}\right) \mathrm{C}=\mathrm{CHCH}_{3}$

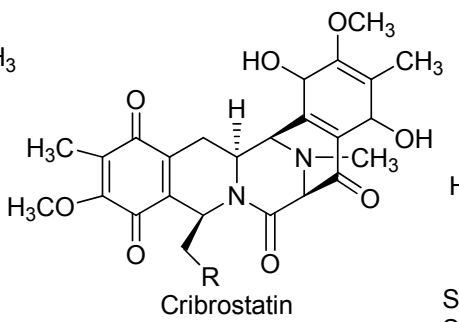

Saframycin $\mathrm{A}, \mathrm{Z}=\mathrm{CN}, \mathrm{R}=\mathrm{CH}_{3} \mathrm{COCONH}$ Saframycin $\mathrm{S}, \mathrm{Z}=\mathrm{OH}, \mathrm{R}=\mathrm{CH}_{3} \mathrm{COCONH}$ Jorumycin, $\mathrm{Z}=\mathrm{OH}, \mathrm{R}=\mathrm{CH}_{3} \mathrm{CO}_{2}$

图 1 化合物 Renieramycin G, Cribrostatin, Saframyeins 和 Ecteinasicdin 743 的结构

Figure 1 Structures of renieramycin G, cribrostatin, saframyeins and cteinasicdin 743

28 国上市, 用于治疗晚期软组织瘤和卵巢癌, 成为第一 个成功开发上市的来源于海洋生物的抗肿瘤药物. 由于 包括 ET-743 在内的这类双四氢异喹啉化合物具有极强 的抗肿瘤活性以及新颖复杂的化学结构和独特的多重 抗肿瘤作用机制, 同时由于自然界中含量极低, 因此这 类双四氢异喹啉天然产物的全合成和结构改造成了新 型抗肿瘤药物研究开发领域的一个热点. 关于这类双四 氢异喹啉天然产物的全合成, 国内外已有多个课题组进 行了深入研究 ${ }^{[7}$ 14], 设计并建立了很多独特的合成策 略, 具有重要的科学价值. 我们小组分别在 2005 年和 2010 年对这类天然抗肿瘤活性双四氢异喹啉生物碱的 全合成研究进行过综述 ${ }^{[15,16]}$, Chen 课题组 ${ }^{[17]}$ 也于 2015 年对此类天然产物的全合成作了一综述. 随着近些年来 对这类双四氢异喹啉天然产物研究的进一步深入, 同时 由于这类天然产物结构的复杂性及全合成路线步骤过 长导致总收率低等原因, 使得对此类天然产物进行结构 改造获得具有相同抗肿瘤活性的结构类似物的研究成 为了一个重要的发展方向. 目前这类双四氢异喹啉结构 类似物的研究主要集中在保留其五并环或五环中的 A、 B、C 三环活性骨架结构的基础上, 对其进行一系列的 结构优化来获得合成路线更简便, 抗肿瘤活性相近或更 优的先导化合物. 本文将就近年来对这类双四氢异喹啉 天然产物类似物的研究进展作以下综述.

\section{1 双四氢异喹啉高级中间体及类似物的合成研 究}

Ecteinascimns 类化合物最早在 1986 年由 Rinehart 课题组 ${ }^{[18,19]}$ 从加勒比海 Ecteinascidia turbinate 海鞘中分 离鉴定得到. 由于其具有多种生物活性和独特的药理作 用机制, 引起了广大生物学家和药学家的研究兴趣, 随 着对该类天然产物研究的逐步深入, 最终促进了用于治 疗晚期软组织瘤和卵巢癌的 ET-743 的成功上市, 使其 成为第一个来源于海洋的抗肿瘤天然药物. 同时, 由于 这类四氢异喹啉天然产物复杂的化学分子结构, 使得 ecteinascidins 类天然产物成为化学全合成研究领域的热
点. 1996 年, Corey 小组 ${ }^{[7]}$ 最先报道了 ET-743 的全合成, 此后 Fukuyama ${ }^{[20]}, \mathrm{Zhu}^{[10]}$, Danishefsky ${ }^{[21]}$ 等相继报道了 ET-743 的全合成研究, 截止目前, 世界上已有多个课题 组报道了该类天然生物碱的全合成研究 ${ }^{[7 \sim 14]}$; 在 ecteinascidins 类似物的合成研究上, 1999 年, Corey 小 组 ${ }^{[3,4]}$ 首先报道了一种 ecteinascidins 类似物 phthalascidin 650 (Pt-650), 该化合物化学结构相对 ET-743 更加简单, 但同 Ecteinascidin-743 具有接近的抗肿瘤活性，并被进 一步作为先导化合物来研究其抗肿瘤活性. 随着对 ecteinascidins 类似物研究的深入, 一些具有较强生物活 性的化合物被化学家和生物学家联合开发出来 ${ }^{[22 ~ 24]}$.

近十年来, Cuevas 课题组 ${ }^{[9]}$ 首先报道了通过 Cyanosafracin B 半合成 ET-743 的研究, 随后该课题 ${ }^{[25]}$ 及 Echavarren 课题组 ${ }^{[26]}$ 又报道了通过 Cyanosafracin B 半合成 ET-743 家族成员及其类似物的研究. 两个课题 组报道的化合物的合成方法类似，该方法是以细菌通过 生化反应产生的大量 Cyanosafracin B 为原料, 进一步合 成 ET-743 及其家族成员和类似物。该方法合成路线简 洁, 可以合成克级的目标产物用来进行进一步的生物活 性研究. 同时, Danishefsky 等 ${ }^{[27]}$ 报道了一种新型的通过 分子内的 Mannich 反应构建五环骨架来合成四氢异喹啉 ecteinascidins 类似物 5. 该类似物的化学结构相对于 ET-743 中的三个四氢异喹啉结构简化为两个四氢异喹 啉结构, 且 A 环和 E 环上的取代基也与天然产物 ET-743 不尽相同, 其化学结构更趋近于 Saframycin 类四氢异喹 啉, 这也使得该化合物的合成路线相对简单, 收率显著 提高. 该化合物的合成路线是以光学纯的四氢异喹啉化 合物 1 和光学纯的芳香丙酸 2 为起始原料, 通过肽键缩 合酰胺生成化合物 $\mathbf{3}$, 化合物 3 再通过脱去酚羟基保护 基及 Dess-Martin 氧化成醛 $\mathbf{4}$, 再通过特异性的 Mannich 反应一步环合成五环骨架化合物 5 (Scheme 1). 2008 年 Danishefsky 等 ${ }^{\left[{ }^{28]}\right.}$ 又在此工作基础上报道了一系列该类 四氢异喹啉 ecteinascidins 类似物的生物活性, 初步的构 效关系研究表明, 该类四氢异喹啉 ecteinascidins 类似物 C-21 位的羰基氰基化，会显著提高其抗肿瘤活性，而对 
<smiles>COc1c(C)c(C)c(OC)c(CC(C(=O)O)N(C)C)c1COc1ccccc1</smiles><smiles>COc1c(C)c(CC(C(=O)O)N2CC(=O)c3cc(C)c(OC)c(OC)c3C2COc2ccccc2)c(OC)c(OC)c1C</smiles><smiles>CCCOC[C@H]1c2c(cc(C)c(OC)c2OC)C(=O)CN1C(=O)C(Cc1c(OC)c(C)c(OC)c(OC)c1OC)[18O]c1ccccc1</smiles>

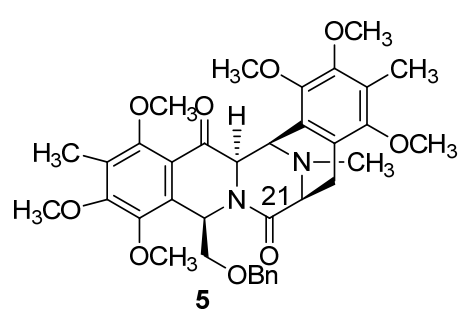

图式 1 化合物 5 的合成

Scheme 1 Synthesis of compound 5

$\mathrm{A}, \mathrm{D}, \mathrm{E}$ 三环的氧化及取代都不会对生物活性有显著影 响. Danishefsky 的研究工作对这类四氢异喹啉 ecteinascidins 类似物构效关系的研究提供了坚实的科学 基础.

Saito 课题组 ${ }^{[29 ~ 35]}$ 在这类四氢异喹啉天然产物的化 学合成及生物活性的研究也进行了数十年系统的工作.
他们开发出了一条独特的合成路线来构建这类四氢异 喹啉天然产物的五并环结构, 通过此方法该课题组合成 了多个这类四氢异喹啉家族的天然产物及类似物 (Scheme 3). 该合成路线是以 2,4,5-三甲氧基-3-甲基苯 甲醛和 1,4-二乙酰基-2,5-哌嗪二酮为起始原料，通过羟 醛缩合并经钯碳还原制得关键中间体 6 , 化合物 6 经氯<smiles>COc1cc(CC2NC(=O)CN(C(C)C)C2=O)c(OC)c(C)c1OC</smiles>

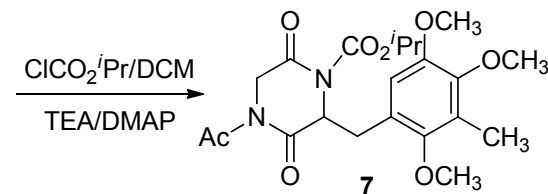<smiles>COc1cc(C=O)c(OC)c(C)c1OC</smiles>
$\mathrm{KOBu}{ }^{t} /{ }^{t} \mathrm{BuOH}, \mathrm{DMF}$<smiles>COc1cc(C=C2NC(=O)C(Cc3cc(OC)c(OC)c(C)c3OC)N(C(=O)O)C2=O)c(OC)c(C)c1OC</smiles><smiles>CC(C)C(C)C(C)C=O</smiles>

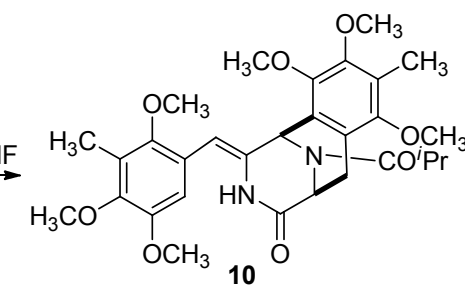
$\underset{\text { (2) } \mathrm{HCHO} / \mathrm{HCOOH}}{\longrightarrow}$

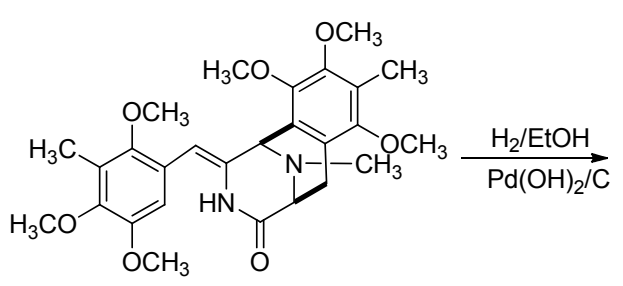<smiles>COc1cc(CC2N[C@@H](C#N)C3Cc4c(OC)c(C)c(OC)c(OC)c4[C@H]2C3C)c(OC)c(C)c1OC</smiles><smiles>CC(C)C(=O)OCc1ccccc1</smiles>

图式 2 化合物 14 的合成

Scheme 2 Synthesis of compound 14 
甲酸异丙酯保护氨基后得化合物 7, 再与 2,4,5-三甲氧 基-3-甲基苯甲醛(8)在碱性条件下发生第二次羟醛缩合 得化合物 9, 化合物 9 先用三叔丁氧基氢化铝锂选择性 地还原 2-位的羰基, 再用甲酸催化环合构建 $\mathrm{C}, \mathrm{D}, \mathrm{E}$ 三环 得化合物 10, 化合物 10 先用三氟乙酸脱去氨基保护基, 再用甲醛对氮原子甲基化得化合物 11, 化合物 11 经氢 化铝锂还原、氰基化得化合物 13, 化合物 13 与苄基保 护的羟基乙醛在三氟乙酸的条件下发生 Pictet-Spengler 反应构建五环骨架得化合物 14. 该课题组后续研究了 在化合物 14 结构基础上的一些类似物的体外抗肿瘤活 性, 发现所合成的类似物的抗肿瘤活性并没有天然产物 本身的活性高, 有的类似物的生物活性甚至显著降低.

2010 年 Lemaire 课题组 ${ }^{[36]}$ 报道了一种 Ecteinascidins 类似物 Pt-622 的合成, 该合成策略采取了汇聚式合成. 以邻苯二酚 15 为原料, 经羟基选择性苄基保护后, Duff 反应得到芳香醛 16, 再对化合物 16 羟基甲基化后与硝 基乙酸乙酯进行 Knoevenagel 缩合成化合物 17, 再经硝 基还原及脱苄基保护得化合物 18; 其次以苯酚 19 为另 一原料，经羟基甲基化、芳基甲基化及 Vilsmeier-Haack 反应得芳香醛 20, 再经 Knoevenagel 缩合得化合物 21,
21 经硝基和酯键还原，伯胺酰基化及成酯得化合物 22 , 化合物 22 经 Bischler-Napieralski 反应得四氢异喹啉 23, 化合物 23 经还原、氨基保护、氧化三步得醛 24, 再将 化合物 18 和 24 经分子间的 Pictet-Spengler 反应得 25, 化 合物 25 经 N-甲基化、仲胺的脱保护基得化合物 26, 化 合物 26 经 swern 氧化、分子内 Strecker 反应关环合成目 标化合物 27 (Pt-622) (Scheme 3). 该课题组在研究这类 四氢异喹啉类似物的构效关系时发现, C-21 位氰基化的 化合物活性要远优于 C-21 位为羰基的化合物，而芳香 环上取代基的变化对活性的影响并不显著.

Fukuyama 课题组 ${ }^{[37]}$ 对这类四氢异喹啉天然产物的 研究主要集中在化学全合成方面，于 2013 年报道了一 种合成 ET-743 时的五环骨架的构建方法, 利用该方法 可以合成一系列的 Ecteinascidin 类似物(Scheme 4). 该 方法是以取代的苯酚 28 为原料, 先用 $\mathrm{PhI}(\mathrm{OAc})_{2}$ 氧化得 二烯酮 29, 再与 $\mathrm{NaCN}$ 反应得化合物腈 30, 然后用苄基 保护酚羟基后，水解氰基得化合物 31，化合物 31 经 Hofmann 重排后水解得胺 32. 其次以来源于天然左旋谷 氨酸的哌嗪二酮 33 为另一起始原料, 先乙酰化其氨基 得化合物 34, 化合物 34 与苯甲醛 35 经 Perkin 缩合得化

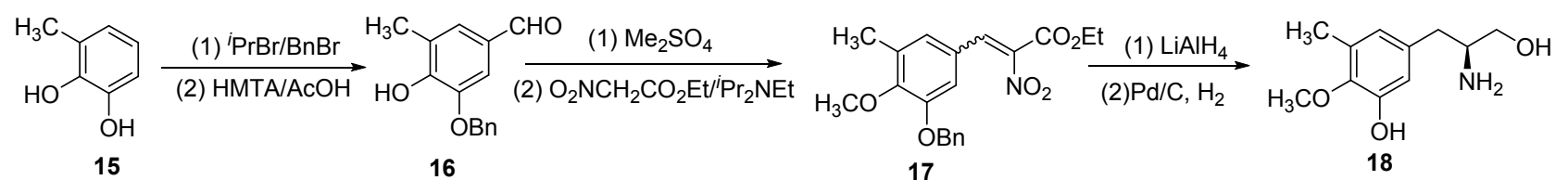<smiles>Oc1ccc2c(c1)OCO2</smiles>

19<smiles>COc1c(C=O)cc2c(c1C)OCO2</smiles>

20<smiles>CCOC(=O)C(=Cc1cc2c(c(C)c1OC)OCO2)C(=O)OCC</smiles>

21<smiles>CCOCCOCCO</smiles>

(2) $\mathrm{PhtCl}$<smiles>COc1c(CC(COC(=O)Cc2ccccc2)NC(=O)c2ccccc2)cc2c(c1C)OCO2</smiles>

22<smiles>COc1c(C)c2c(c3c1OCO3)C(Cc1ccccc1)N[C@H](COC(=O)Cc1ccccc1)C2</smiles>

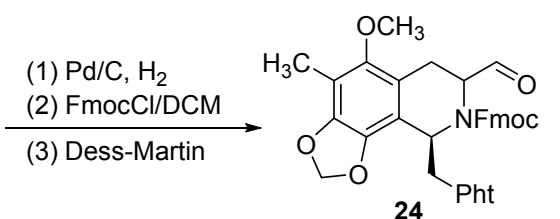

18, TFA

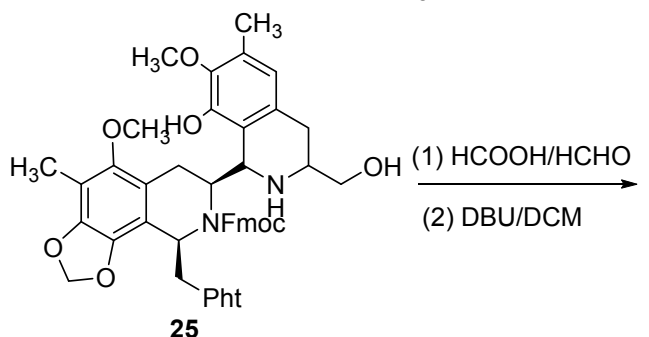<smiles>COc1c(C)cc2c(c1OC)[C@@H](C1Cc3c(OC)c4c(c(C)c3C(C[PH])CN1C)OCO4)N(C)C(CO)C2</smiles>

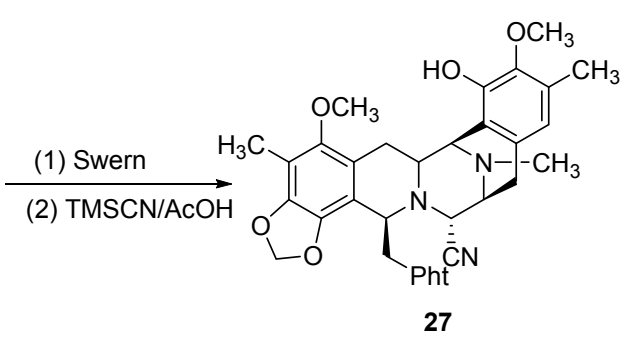

图式 3 化合物 27 (Pt-622)的合成

Scheme 3 Synthesis of compound 27 (Pt-622) 


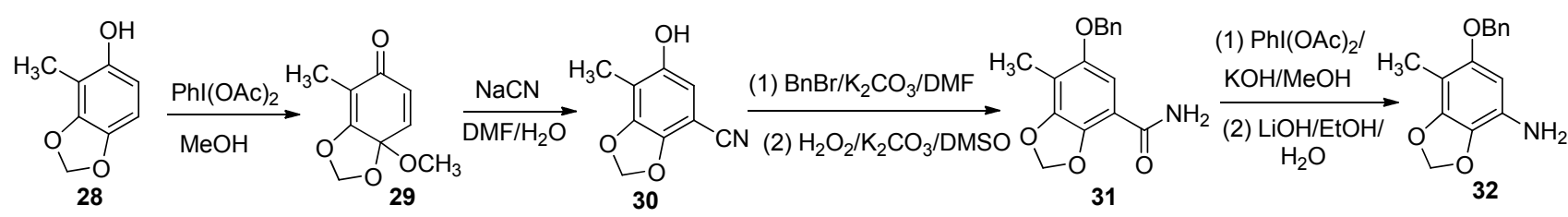

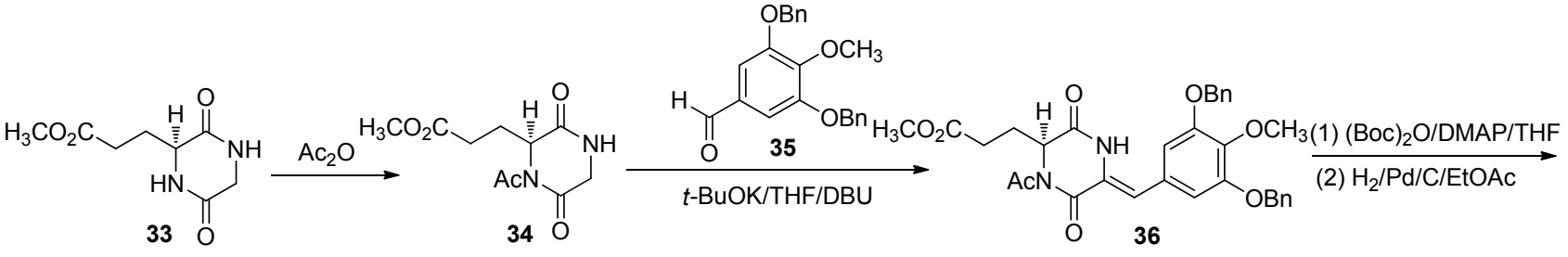

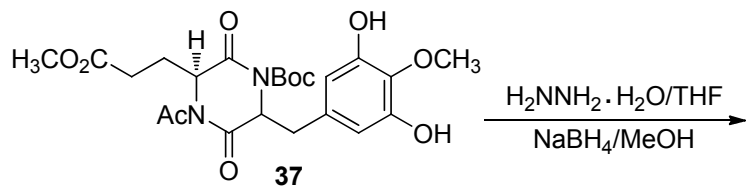<smiles>COc1c(O)cc(CC2C(=O)N[C@H](CCC(=O)O)C(O)[C@H]2O)cc1O</smiles>

$\underset{\text { (1) } \mathrm{TFA} / \mathrm{CF}_{3} \mathrm{CH}_{2} \mathrm{OH}}{\stackrel{\mathrm{PhNf}}{2} / \mathrm{DMAPI}}$

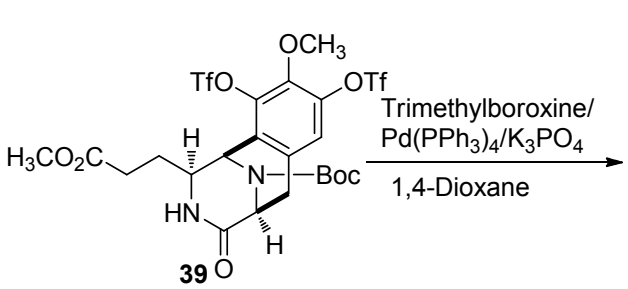

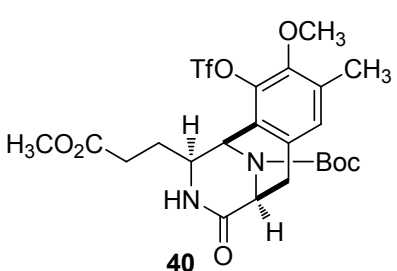

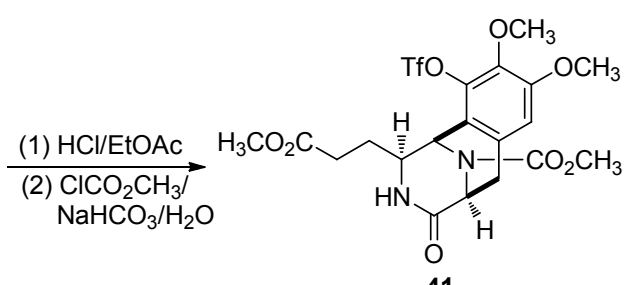

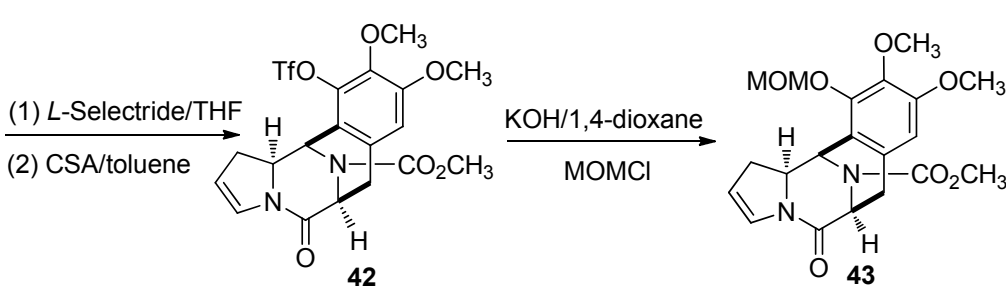<smiles>Cc1c(OCc2ccccc2)cc(N)c2c1O[13CH]O2</smiles>

41 (1) $\mathrm{BF}_{3} \cdot \mathrm{OEt}_{2} / t-\mathrm{BuONO} / \mathrm{TH}$ $\underset{\substack{\text { (2) } \mathrm{Pd}_{2}(\mathrm{dba})_{3} / \mathrm{NaOAcl} \\ \mathrm{MeCN} / \mathrm{THF}}}{\longrightarrow}$<smiles>Cc1c(OCc2ccccc2)cc([C@H](C)NC(=O)C(C)C)c2c1OCO2</smiles>

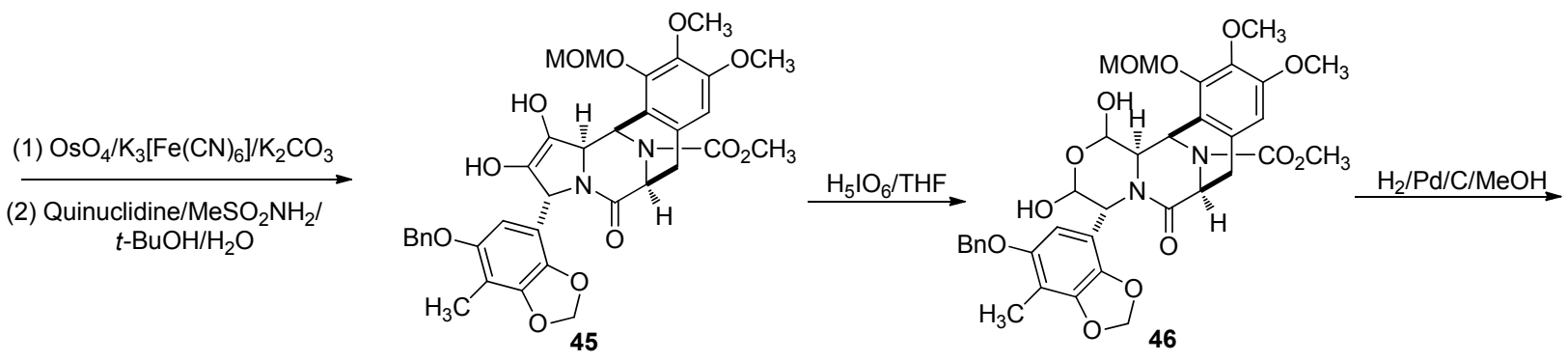

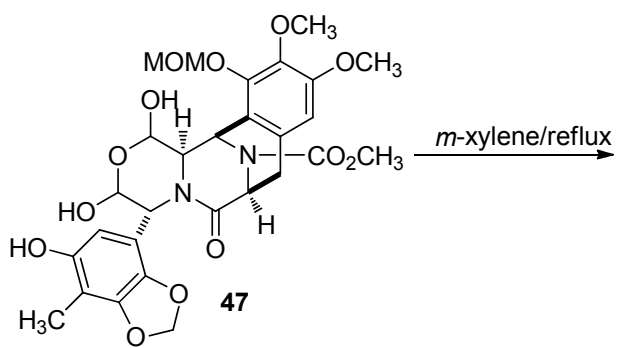

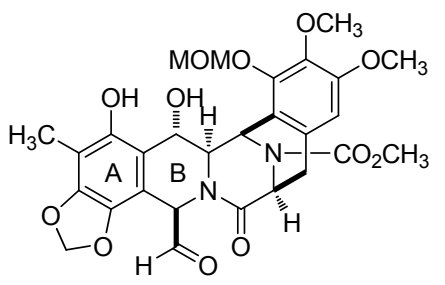

图式 4 五环骨架化合物 48 的合成

Scheme 4 Synthesis of pentacyclic skeleton compound 48 
合物 36, 再用 Boc 酸酐保护化合物 36 内酰胺上的氨基, 然后催化氢化脱除酚羟基上的茮基的同时还原烯键得 化合物 37, 化合物 37 用水合肼脱除氨基上的乙酰基后, 用 $\mathrm{NaBH}_{4}$ 选择性地还原内酰胺上的羰基得化合物 $\mathbf{3 8}$, 化合物 38 在 TFA 的作用下, 经酰亚胺离子中间态介导 进行环合反应, 再用 $\mathrm{PhNTf}_{2}$ 对酚羟基进行三氟甲磺酸 化得化合物 39. 化合物 39 与三甲基环三㸴氧烷在三苯 基磷的催化下选择性地发生 Suzuki-Miyaura 反应得化合 物 40, 再将化合物 40 的 Boc 保护基转换成甲氧羰基保 护基得化合物 41. 化合物 41 用三仲丁基嗍氢化锂还原 酯键成醛基后与氨基反应得半缩醛胺中间态, 再用右旋 樟脑磺酸催化经脱水反应得稀酰胺 42. 将化合物 42 中 酚羟基的三氟甲磺酸保护基用甲氧甲基替换得化合物 43. 化合物 32 在亚硝酸丁酯的作用下形成重氮盐并与 烯酰胺 43 在钯催化下发生 Heck 反应得化合物 44, 这是 该五环骨架构建方法最关键的一步反应。化合物 44 以 $\mathrm{K}_{3}\left[\mathrm{Fe}(\mathrm{CN})_{6}\right]$ 作为共氧化剂用四氧化锇氧化双键得 1,2-二 醇化合物 $\mathbf{4 5}$, 化合物 45 用 $\mathrm{H}_{5} \mathrm{IO}_{6}$ 氧化成双醛的过渡态, 再经分子内的缩合脱水得化合物 46, 化合物 46 经催化 氢化脱除苄基得化合物 47, 化合物 47 在二甲苯中回流 反应重新裂解为二醛过渡态, 并与富电子的 $\mathrm{A}$ 环环合形 成五环骨架化合物 48. 该合成方法较为复杂, 整体收率 不高, 但该方法有别于传统的利用该类四氢异喹啉仿生 合成策略通过 Pictet-Spengler 反应来构建五环骨架, 而 是通过 Heck 反应得到关键中间体 44 来构建五环骨架, 其策略十分新颖。此法可用来合成 A 环具有不同取代
基的衍生物, 为该类化合物构效关系的研究开辟了新的 方向.

陈小川课题组 ${ }^{[38 \sim 42]}$ 也对此类四氢异喹啉生物碱进 行了研究. 该课题组开发的合成方法较以往其他课题组 报道的方法在收率上有明显提高. 该方法以天然 $L$-酪氨 酸为原料，通过羟甲基化/离子型氢化和甲酰化 /Baeyer-Villiger 氧化等 8 步反应依次在苯环上引入甲基 和羟基得到三取代苯丙氨醇 49, 化合物 49 与苄氧基乙 醛发生高选择性的 Pictet-Spengler 等三步反应得四氢异 喹啉 50, 化合物 50 经 Swern 氧化得四氢异喹啉醛 51. 化 合物 51 与同样以 $L$-酪氨酸为原料经八步反应得到的化 合物 52 发生第二次 Pictet-Spengler 反应得异构体 $\mathbf{5 3}$ 和 54. 化合物 54 经 $N$-甲基化、脱 Boc 保护基得化合物 56, 化合物 56 经分子内的 Strecker 反应成功地构建了五环 骨架化合物 57. 该合成方法较以往的合成路线在收率 上有较大的提高，可将该方法开发成具有一定应用价值 的工艺路线, 为该类四氢异喹啉天然产物及衍生物深入 的生物学研究提供足量的样品(Scheme 5).

\section{2 双四氢异喹啉简化物的合成研究}

由于包含 Ecteinasicdin743 在内的这类四氢异喹啉 生物碱复杂的化学结构以及优良的生物活性，使得对其 进行结构简化和构效关系的研究也成为了该类四氢异 喹啉天然产物的一个重要研究内容. 世界范围内多个课 题组对此开展了相关研究, 其中, Avendańo 课题组在近 十年间陆续报道了 Ecteinasicdins 类生物碱简化物的合

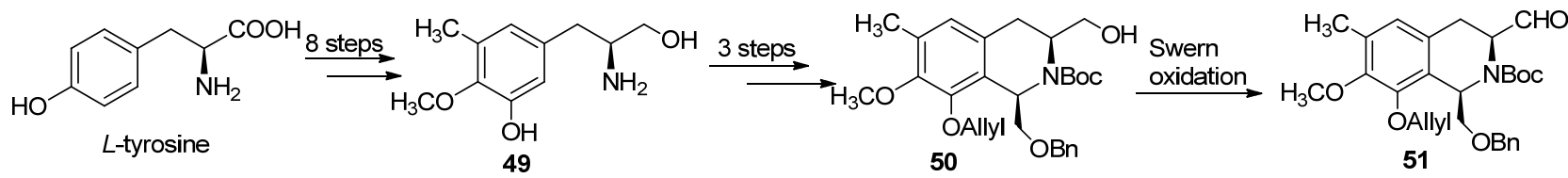

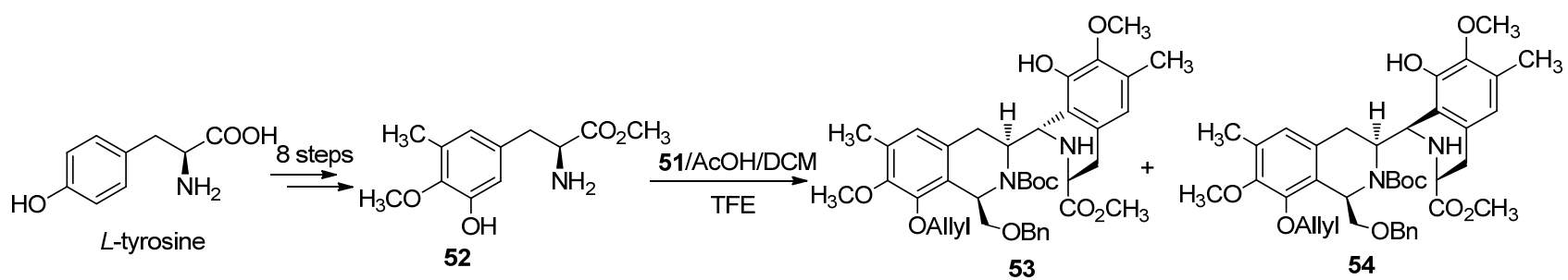

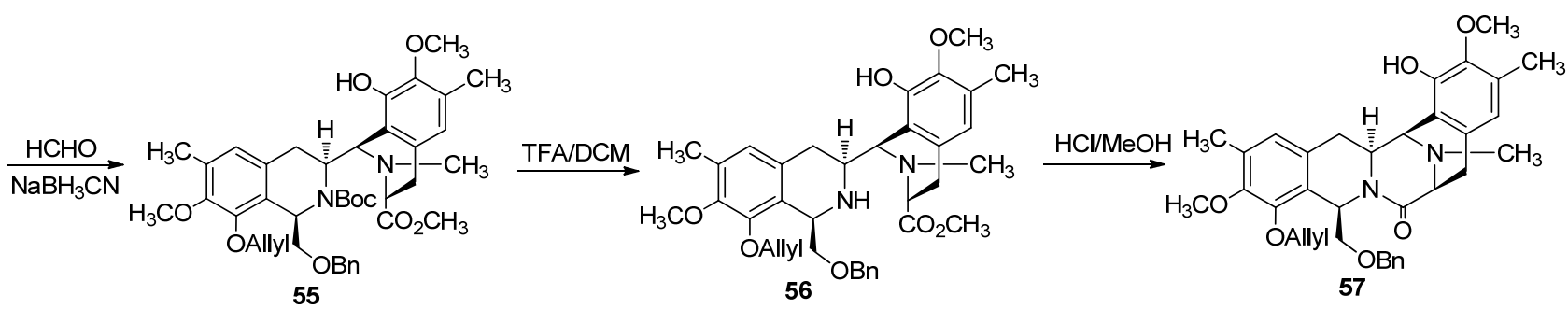

图式 5 五环骨架化合物 $\mathbf{5 7}$ 的合成

Scheme 5 Synthesis of pentacyclic skeleton compound 57 
成及活性研究. 该课题组 ${ }^{[43]}$ 在 2007 年报道了合成五环 骨架类似物时发现了一个三环体系的中间体的活性反 而优于五环骨架结构的简化物, 并于 2008 年 ${ }^{[44]}$ 后续报 道了一系列该类简化物的活性研究情况. 通过对三环体 系中的 C-21 位氰基化和 C-22 位取代基的变化衍生得到 的一系列简化物的活性研究, 发现了一个先导化合物 59b. 由于这类简化物的结构特点是仅保留了五环骨架 中的 $\mathrm{A}, \mathrm{B}, \mathrm{C}$ 三环体系, 该类简化物的合成方法较天然产 物的合成更为简捷, 成药可能性也显著提高. 这类三环 体系简化物的合成方法是以经典方法中的 1,4-哌嗪二酮 58 为起始原料, 通过分子间的 Pictet-Spengler 反应构建 三环骨架的简化物 59 60 (Scheme 6).

在后期的研究中, Avendańo 课题组 ${ }^{[45 ~ 47]}$ 陆续报道 了一类新型三环体系简化物的活性研究情况. 该类简化 物的结构特点主要是对化合物 59b 三环骨架结构的 N-2,
C-1, C-3 和 C-15 位的不同取代而衍生的一系列衍生物, 并系统地研究了其与五环骨架间的活性对比. 遗憾的是 该次研究并没有发现比 59b 活性更好的化合物, 但研究 发现 N-2 位的酰基化能提高三环体系的活性, C-6 位的 苄基化也能显著提高活性，而在 C-3 位引入芳环却会降 低三环体系的活性, 在 C-11 和 C-12 位之间或在 C-1 和 C-12 位之间引入不饱和键也会降低活性.

另外, 在 Avendańo 的研究基础上, Kimpe ${ }^{[48]}$ 于 2014 年报道了一类仅保留了该类四氢异喹啉天然产物五环 骨架中的 $\mathrm{A}, \mathrm{B}, \mathrm{C}$ 三环体系的简化物. 这类简化物是以 $N$-(二苯基亚甲基)甘氨酸乙酯(64)和溴甲基取代的苯衍 生物 65 为起始原料(Scheme 7), 在水和二氯甲烷的非均 相体系中用相转移催化剂四丁基硫酸氢铵的作用下缩 合并酸解得化合物 66, 化合物再经过 Pictet-Spengler 反 应生成四氢异喹啉化合物中间体 67 , 然后用氯乙酰氯

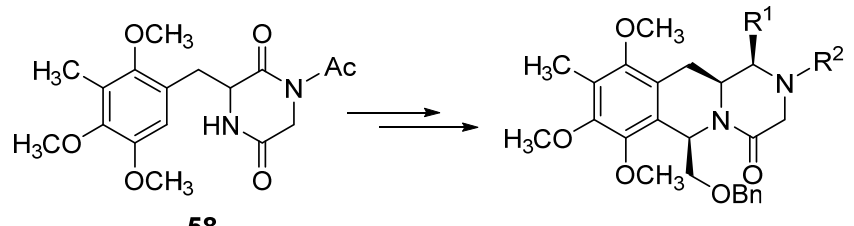<smiles>[R]C1C2Cc3c(OC)c(C)c(OC)c(OC)c3C(CO)N2C(=O)C(C[Al])N1[R]</smiles>
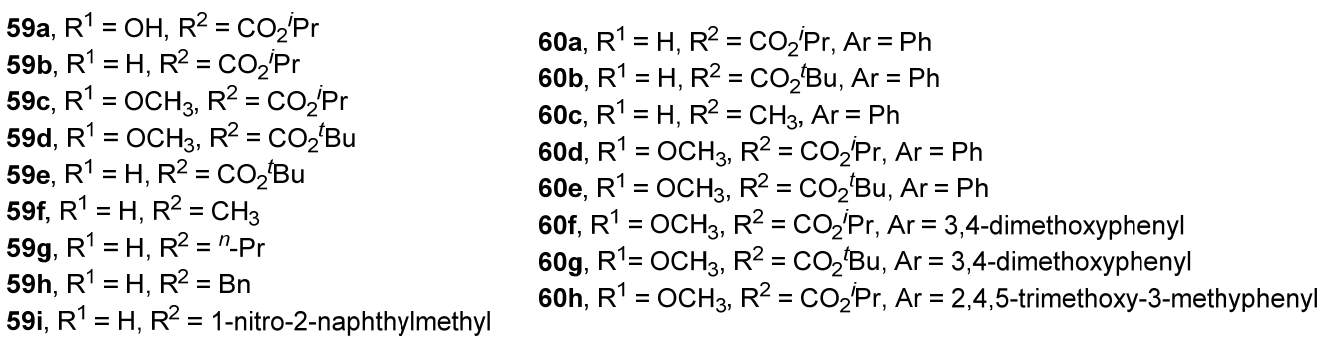<smiles>[R]C1C2Cc3c(OC)c(C)c(OC)c(OC)c3C(CO)N2C(=O)[C@H](CBr)N1[R]</smiles>

61, $\mathrm{R}^{1}=\mathrm{OCH}_{3}, \mathrm{R}^{2}=\mathrm{CO}_{2}{ }^{\mathrm{P}} \mathrm{Pr}, \mathrm{Ar}=$ 2,4,5-trimethoxy-3-methyphenyl<smiles>[R]C[C@H]1c2c(c(OC)c(C)c(OC)c2OC)C=C2CN[C@H](C[Te])C(=O)N21</smiles>

62a, $\mathrm{R}=\mathrm{Bn}, \mathrm{Ar}=\mathrm{Ph}$

62b, $R=$ naphthylcarbonyl $\mathrm{Ar}=2,4,5$-trimethoxy-3-methyphenyl<smiles>[R]CC1c2c(c(OC)c(C)c(OC)c2OC)CC2=CN[C@H](C[12OH])C(=O)N21</smiles>

63, $R=$ cinnamoyl

A $r=2,4,6=$ trimethoxy-3-methylphenyl

图式 6 三环骨架化合物的合成

Scheme 6 Synthesis of tricyclic skeleton compounds<smiles>CCC(=O)CN=C(c1ccccc1)c1ccccc1</smiles><smiles>[R6]c1cc(CBr)c(OC)c([R])c1[R]</smiles>

(1) $\mathrm{KOH} / \mathrm{Bu}_{4} \mathrm{NHSO}_{4} / \mathrm{DCM}$

(2) $\mathrm{HCl} / \mathrm{THF}$<smiles>[R]c1c(OC)cc(CC(N)C(=O)OCC)c(OC)c1[R]</smiles>

$\underset{\text { (2) } \mathrm{TFA} \mathrm{HCHO} / \mathrm{H}_{2} \mathrm{O}}{\longrightarrow}$<smiles>[R]c1c([R])c(OC)c2c(c1OC)CC1C(=O)N([R])CC(=O)N1C2</smiles>

图式 7 化合物 69 的合成

Scheme 7 Synthesis of compound 69 
对仲胺进行酰化后得化合物 68 , 化合物 68 进一步与取 代伯胺环合生成六氢哌嗪并 $[1,2-b]$ 异喹啉类化合物 69. 该合成方法较为新颖, 但该课题组并没有进一步报道这 类简化物的活性研究结果.

我们小组自 1999 年以来对这类双四氢异喹啉天然 产物的化学合成及生物活性也进行了深入的研究, 目前 我们已成功的建立了一种立体专一构建这类双四氢异 喹啉五环骨架的技术方法 ${ }^{[49]}$, 不仅成功地全合成了 (-)-renieramycin G、(一)-Jorumycin、(-)-Saframycin A 及其衍生物 ${ }^{[50-54]}$, 而且也将该技术方法成功地应用到 对这类生物碱简化物的合成研究中，合成了一系列的结 构简化物, 同时系统地研究了这些简化物的体外抗肿瘤 活性和构效关系 ${ }^{[55 \sim 58]}$

我们的合成策略是以光学纯的 $L$-dopa 为起始原料, 分别合成四氢异喹啉 70 和苯丙氨酸 71 两个结构单元 (Scheme 8), 利用肽键将它们连接起来构建关键中

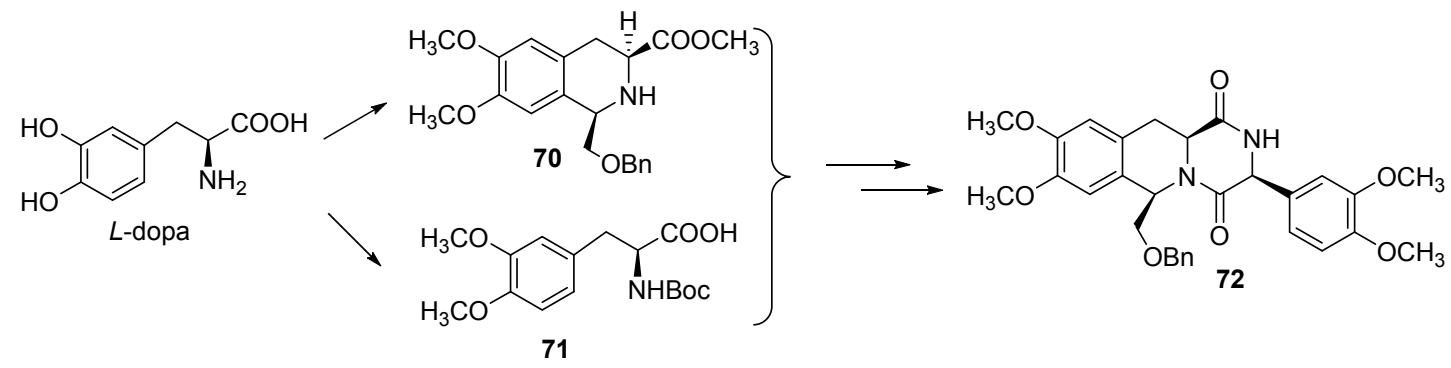

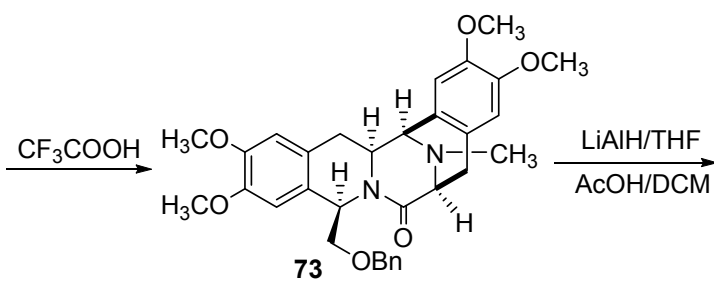<smiles>[Z2]CC(I)c1cc(OC)c(OC)cc1CCI</smiles><smiles>COc1cc2c(cc1OC)[C@H]1[C@@H](C)[C@@H](C#N)NC[C@H]2[C@@H]1C(C)C</smiles>

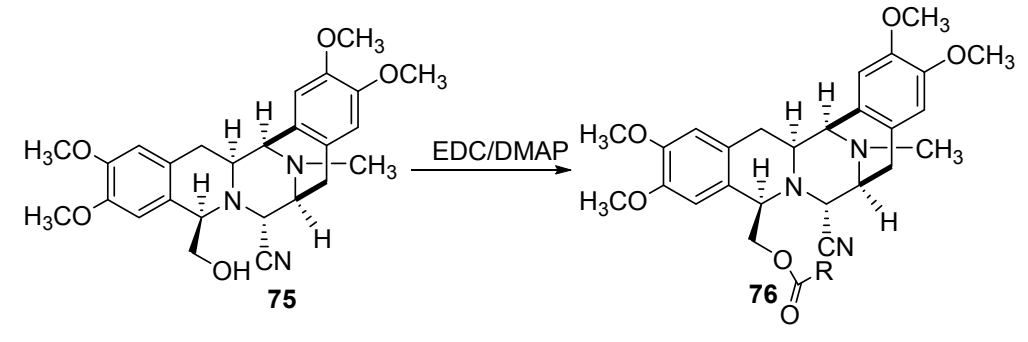

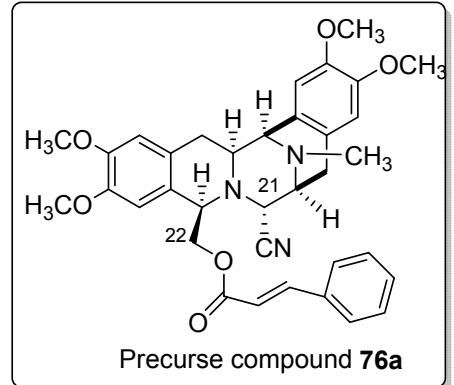

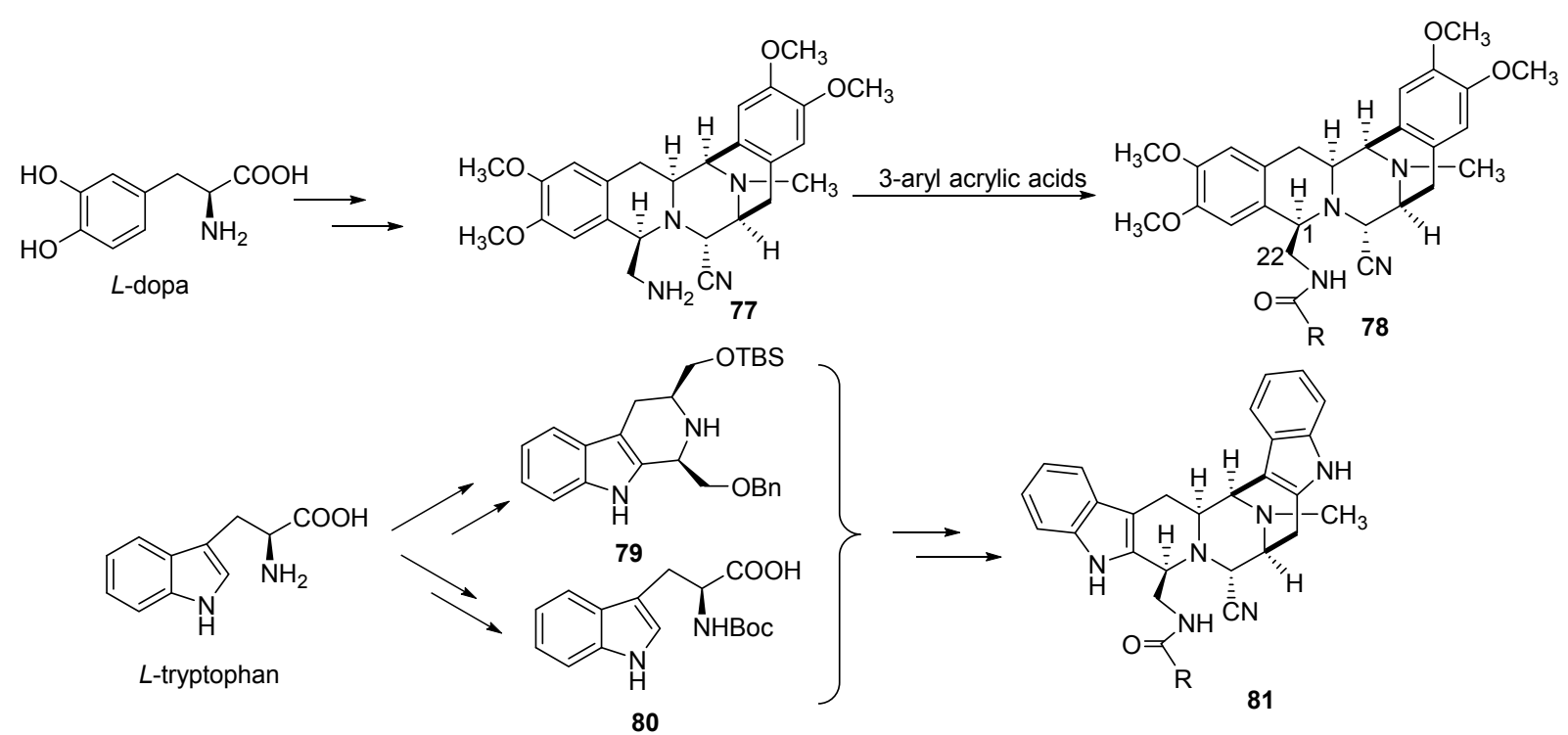

图式 9 化合物 76a、78 和 81 的合成

Scheme 9 Synthesis of compounds 76a, 78 and 81 
间体哌嗪二酮 72, 这是本路线的关键步骤. 然后通过改 良的 Pictet-Spengler 反应实现分子内环合, 形成五环骨 架化合物 73, 最后将 C-21 位的羰基转化成氰基, 得到 目标化合物 74. 我们用较少的步骤完成了五环的构建, 同时确立了分子中的四个手性中心，总收率达到 $12 \%$ (Scheme 8). 该路线步骤较短、操作方便、反应条件温 和、收率较高. 光学纯的目标化合物 74 经过进一步的结 构修饰得到了一系列双四氢异喹啉的结构简化物 76 . 在笁选了其活性的基础上系统评价了该类化合物的构 效关系, 发现该类化合物的五环骨架为其活性必需结 构, C-21 位为氰基的化合物的生物活性要好于非氰基的 化合物, 而对 C-22 位的结构修饰能对活性有较大的影 响. 通过进一步的研究, 我们发现了一个先导化合物 $76 \mathbf{a}$, 通过初步的构效关系研究发现, 其 C-22 位侧链的 改变对其活性的影响较为明显, 在此构效关系研究的基 础上, 我们对先导化合物 76a 进行了进一步的结构优化, 分别设计并合成了两类具有较好抗肿瘤活性的新型结 构类似物 ${ }^{[56,57]}$. 该路线是以 $L-d o p a$ 为起始原料, 通过构 建五环骨架化合物 77, 再通过对化合物 77 的 C-22 位氨 基进行侧链衍生化得到系列目标化合物 78(Scheme 9), 通过体外活性测试发现这两类简化物均具有较强的抗 肿瘤活性. 同时, 我们根据该类类似物的结构特点, 创 新性的以天然氨基酸 L-tryptophan 为手性原料设计并合 成了一类吲哚并哌嗪环结构的新型化合物 $\mathbf{8 1}^{[58]}$ (Scheme 9), 并通过体外抗肿瘤活性测试显示其具有良 好的抑制肿瘤细胞的活性. 以上工作为后期对该类类似 物构效关系进一步的深入研究和为寻找到具有潜在抗 肿瘤活性的临床候选药物奠定了基础.

\section{3 结束语}

至 1974 年 Kluepfel 等从 Streptomyces lusitanus 中分 离到第一个具有生物活性的四氢异喹啉天然产物开始, 全世界众多课题组对此类四氢异喹啉天然产物进行了 广泛而深入的研究，不仅在其药理作用机制方面取得了 重要进展, 而且在其化学合成领域进行了系统的研究, 推动了 ET-743 作为第一个来源于海洋的治疗晚期软组 织瘤和卵巢癌的天然产物的成功上市, 也进一步推动了 以天然产物作为临床药物来源的科学研究. 目前, 关于 这类天然四氢异喹啉的化学全合成及半合成的研究都 取得了显著的成果, 如最近由美国加州理工学院的 Stlotz $^{[59]}$ 课题组在 Science 上报道的一篇关于 (一)-Jorumycin 的全合成工作使得这类双四氢异喹啉天 然分子再一次吸引了全球化学家的目光, 但这类双四氢 异喹啉天然产物的结构类似物和简化物以及构效关系 的研究还略显单薄, 而结构更加简单、合成过程更加简
洁、毒副作用更少、生物活性更加优良的类似物及简化 物依然是目前天然产物进一步开发成临床用药的关键 所在. 同时，在此类双四氢异喹啉天然产物药理活性的 多样性及药理作用靶点还没有完全研究清楚的基础上, 仍然需要我们进一步不断的研究来充实.

\section{References}

[1] Kluepfel, D.; Baker, H. A.; Piattoni, G.; Sehgal, S. N.; Sidorowicz, A.; Singh, K.; Vezina, C. J. Antibiot. 1975, 28, 497.

[2] Scott, J. D.; Williams, R. M. Chem. Rev. 2002, 102, 1669

[3] Martinez, E. J.; Owa, T.; Schreiber, S. L.; Corey, E. J. Proc. Natl. Acad. Sci. U. S. A. 1999, 96, 3496.

[4] Martinez, E. J.; Corey, E. J.; Owa, T. Chem. Biol. 2001, 8, 1151.

[5] Plowright, A. T.; Schaus, S. E.; Myers, A. G. Chem. Biol. 2002, 9 , 607.

[6] Cuevas, C.; Manzanares, I.; Perez, M.; Martin, M. J.; Rodriguez, A.; Munt, S. (Pharma Mar, S. A.). ES 2231486 T3, 2003.

[7] Corey, E. J.; Gin, D. Y.; Kania, R. S. J. Am. Chem. Soc. 1996, 118 , 9202.

[8] Eduardo, J. M.; Corey, E. J. Org. Lett. 1999, 1, 75.

[9] Cuevas, C.; Pérez, M.; Martín, M. J.; Chicharro, J. L.; Fernádez, R. C.; Flores, M.; Francesch, A.; Gallego, P.; Zarzuelo, M.; Calle, F.; García, J.; Polanco, C.; Rodríguez, I.; Manzanares, I. Org. Lett. 2000, 2, 2545 .

[10] Chen, J.-C.; Chen, X.-C.; Michele, B. C.; Zhu, J.-P. J. Am. Chem. Soc. 2006, 128,87

[11] Chen, X.; Zhu, J. Angew. Chem., Int. Ed. 2007, 46, 3962.

[12] Jonathan, W.; Chen, Y.-Y.; Williams, R. M. J. Am. Chem. Soc. 2005, $127,12684$.

[13] Smith, L. H. S.; Nguyen, T. T.; Sneddon, H. F.; Procter, D. J. Chem. Commun. 2011, 47, 10821

[14] Xu, S.-H.; Wang, G.; Zhu, J.-J.; Shen, C.; Yang, Z.-Z.; Yu, J.; Li, Z.; Lin, T.-H.; Sun, X.; Zhang, F.-L. Eur. J. Org. Chem. 2017, 5, 975.

[15] Wang. Y.; Tang, Y.-F.; Liu, Z.-Z.; Chern, S.-Z.; Liang, X.-T. Chin. J. Org. Chem. 2005, 25, 42 (in Chinese).

(王晔, 唐叶峰, 刘站柱, 陈世智, 梁晓天, 有机化学, 2005, 25, 42.)

[16] Liao, X,-W.; Dong, W.-F.; Liu, W.; Chen, S.-Z.; Liu, Z.-Z. Chin. J. Org. Chem. 2010, 30, 317 (in Chinese).

(廖祥伟，董文芳，刘伟，陈世智，刘站柱，有机化学， 2010，30, 317.)

[17] Song, Y.-T.; Hu, L.-L.; Chen, R.-J.; Chen, X.-C. Chin. J. Org. Chem. 2015, 35, 1627 (in Chinese). (宋玉廷，胡玲玲，陈瑞蛟，陈小川，有机化学, 2015, 35, 1627.)

[18] Rinehart, K. L.; Holt, T. G.; Fregeau, N. L.; Stroh, J. G.; Kieffer, P. A.; Sun, F.; Li, L.-H.; Martin, D. G. J. Org. Chem. 1990, 55, 4512.

[19] Rinehart, K. L.; Holt, T. G.; Fregeau, N. L.; Stroh, J. G.; Kieffer, P. A.; Sun, F.; Li, L.-H.; Martin, D. G. J. Org. Chem. 1991, 56, 1676.

[20] Endo, A.; Yanagisawa, A.; Abe, M.; Tohma, S.; Kan, T.; Fukuyama, T. J. Am. Chem. Soc. 2002, 124, 6552.

[21] Zheng, S.; Chan, C.; Furuuchi, T.; Wright, B.; Zhou, B.; Guo. J.; Danishefsky, S. J. J. Angew. Chem., Int. Ed. 2006, 45, 1754.

[22] Jeffrey, R..; Martin, S.; Jason, O.; Peter, S.; Stacie, A. D.; Catherine, M.; Hyunjin, M. K.; Zhang, P-L.; Neil, S.; Katherine, G. M.; Juthamas, S.; Doris, G.; Ohn, E.; Peter, R. Y.; Myers, A. G.; Green, M. J. Bioorg. Med. Chem. Lett. 2006, 16, 4884.

[23] Ocio, E. M.; Maiso, P.; Chen, X.; Garayoa, M.; Alvarez-Fernandez, S.; SanSegundo, L.; Vilanova, D.; Lopez-Corral, L.; Montero, J. C.; HernandezIglesias, T.; Alava, E.; Galmarini, C.; Aviles, P.; Cuevas, C.; Pandiella, A. Blood. 2009, 113, 3781.

[24] Leal, J. F. M.; Garcia-Hernandez, V.; Moneo, V.; Domingo, A.; Bueren-Calabuig, J. A.; Negri, A.; Gago, F.; Guillen-Navarro, M. J.; Aviles, P.; Cuevas, C.; GarciaFernandez, L. F.; Galmarini, C. Biochem. Pharmacol. 2009, 78, 162.

[25] Roberto, M.; Martinez, V.; Alberto, R.; Natividad, R.; Flores, M.; 
Gallego, P.; Manzanares, I.; Cuevas, C. J. Org. Chem. 2003, 68, 8859 .

[26] Plácido, A. C.; Marta, P.; Cuevas, C.; Andrés, F.; Manzanares, I.; Antonio, M.; Echavarren. Eur. J. Org. Chem. 2006, 8, 1926.

[27] Zhou, B.-S.; Guo, J. S.; Danishefsky, S. J. Tetra. Lett. 2000, 41, 2043.

[28] Benjamin, J.; Wright, D.; Collin C.; Danishefsky, S. J. J. Nat. Prod. 2008, 71, 409 .

[29] Kubo, A.; Saito, N.; Yamauchi, R.; Sakai, S. Chem. Pharm. Bull. 1987, 35, 2158 .

[30] Kubo, A.; Saito, N.; Yamato, H.; Kawakarmi, Y. Chem. Pharm. Bull. 1987, 35, 2525.

[31] Kubo, A.; Saito, N.; Nakamura, M.; Ogata, K.; Sakai, S. Heterocycles 1987, 26, 1765 .

[32] Kubo, A.; Saito, N.; Yamato, H.; Yamauchi, R.; Hiruma, K.; Inoue, S. Chem. Pharm. Bull. 1988, 36, 2607.

[33] Kubo, A.; Saito, N.; Yamato, H.; Masubuchi, K.; Nakamura, M. J. Org. Chem. 1988, 53, 4295.

[34] Shinya, K.; Shintaro, K.; Masayuki, A.; Yoshifumi, U.; Koizumi, Y.; Masashi, Y.; Saito, N. Heterocycles 2015, 90, 327.

[35] Masashi, Y.; Ryoko, T.; Toshihiro, S.; Vy, H. L.; Williams, R. M.; Saito, N. J. Org. Chem. 2016, 81, 4039.

[36] Christian, R.; Razafindrabe.; Sylvain, A.; Benjamin B.; Andriantsiferana, M.; Stéphane, P. R.; Lemaire, M. Tetrahedron 2010, 66, 9061 .

[37] Kawagishi, F.; Toma, T.; Inui, T.; Yokoshima, S.; Fukuyama, T. J. Am. Chem. Soc. 2013, 135, 13684

[38] Chen, R.; Liu, H.; Chen, X. J. Nat. Prod. 2013, 76, 1789.

[39] Chen, R.; Zhu, D.; Hu, Z.; Zheng, Z.; Chen, X. Tetrahedron: Asymmetry 2010, 21, 39

[40] Chen, R.; Liu, H.; Liu, X.; Chen, X. Tetrahedron 2013, 69, 3565.

[41] Liu, H.; Chen, R.; Chen, X. Org. Biomol. Chem. 2014, 12, 1633.

[42] Jia, J.; Chen, R.; Liu, H.; Li, X.; Jia, Y.; Chen, X. Org. Biomol. Chem. 2016, 14, 7334 .

[43] González, J. F.; Cuesta, E.; Avendano, C. Bioorg. Med. Chem. 2007,
$15,112$.

[44] Ortín, I.; González, J. F.; Cuesta, E.; Cristina, M. G.; Rosario P.; Avendano, C. Bioorg. Med. Chem. 2008, 16, 9065.

[45] Ortín, I.; González, J. F.; Cuesta, E.; Avendano, C. Tetrahedron 2009, 65, 2201.

[46] Ortín, I.; González, J. F.; Cuesta, E.; Avendano, C. Tetrahedron 2009, 65, 9944.

[47] Ortín, I.; González, J. F.; Cuesta, E.; Avendano, C. Bioorg. Med. Chem. 2010, 18, 6813.

[48] Tuyen, N. V.; Pieter, C.; Norbert, D. K. Synlett 2014, 45, 69.

[49] Tang, Y.-F.; Liu, Z.-Z.; Chen, S.-Z. Tetrahedron Lett. 2003, 44, 7091 .

[50] Liao, X.-W.; Liu, W.; Dong, W.-F.; Guan, B.-H.; Chen, S.-Z.; Liu, Z.-Z. Tetrahedron 2009, 65, 5709.

[51] Liu, W.; Dong, W.-F.; Liao, X.-W.; Yan, Z.; Guan, B.-H.; Wang, N.; Liu, Z.-Z. Bioorg. Med. Chem. Lett. 2011, 21, 1419.

[52] Dong, W.-F.; Liu, W.; Liao, X.-W.; Guan, B.-H.; Chen, S.-Z.; Liu, Z.-Z. J. Org. Chem. 2011, 76, 5363

[53] Liu, W.; Liao, X.-W.; Dong, W.-F.; Yan, Z.; Wang, N.; Liu, Z.-Z. Tetrahedron 2012, 68, 2759

[54] Dong, W.-F.; Liu. W.; Yan, Z.; Liao, X.-W.; Guan, B.-H.; Wang, N.; Liu, Z.-Z. Eur. J. Med. Chem. 2012, 49, 239.

[55] Liu, Z.-Z.; Wang, Y.; Tang, Y.-F.; Chen, S.-Z.; Chen, X.-G.; Li, H.-Y. Bioorg. Med. Chem. Lett. 2006, 16, 1282.

[56] Guo, J.; Dong, W.-F.; Liu, W.; Yan, Z.; Wang, N.; Liu, Z.-Z. Eur. J. Med. Chem. 2013, 62, 670 .

[57] Guo, J.; Yang, Y.; Wang, N.; Liu, Z.-Z. Tetrahedron Lett. 2018, 33, 3202.

[58] Lu, X.-R.; Pan, X.; Yang, Y.; Ji, M.; Chen, X.-G.; Xiao, Z.-Y.; Liu, Z.-Z. Eur. J. Med. Chem. 2017, 135, 260.

[59] Eric, R. W.; Aurapat, N.; Max, K.; Guillaume, L.; Gerit, M. P.; Martina, S. J. M.; Dylan, C.; Christopher, D. G.; Pamela, M. T.; Christopher, K. H.; Kenji, N.; Emil, G.; Christian, U. G.; Kevin, M. A.; Scott, C. V.; Dennis, J. S.; Brian, M. S. Science 2019, 363, 270. 